\title{
Some remarks about quantum diffusion for Hubbard models
}

\author{
Rémy Mosseri \\ Groupe de Physique des Solides, CNRS et Universités \\ Paris 7 et 6, Tour 23, 2 place Jussieu \\ 75251 Paris Cedex 05, France \\ e-mail: mosseri@gps.jussieu.fr
}

November 23, 2018

\begin{abstract}
Some exact results are given, that connect, for a general magnitude of the interaction term $U$, the repulsive and attractive Hubbard model, in terms of the eigenspectra and quantum diffusion properties. In particular, it is shown that, for some initial conditions, the quantum evolution cannot differenciate between the attractive and repulsive models. These results apply to both fermionic and bosonic models, in any dimension and for general filling, as far as the underlying structure is bipartite.
\end{abstract}

\section{Introduction}

Among the numerous models that have been studied for years to describe correlated systems, the most popular is probably the Hubbard model[1], parametrised by a first-neighbor hopping term $\beta$ and on-site interaction energy $U$. The particles, electrons or bosons, can hop between orbitals located at neighbouring sites of a $d$-dimensional lattice. In one dimension, the exact eigenspectrum, for spin $\frac{1}{2}$ electrons, is known, thank to the well-known solution by Lieb and $\mathrm{Wu}$ [2]. In higher dimension, no exact solution has been found, despite the large efforts intended to fulfil this task[3]. Therefore, partial results are welcomed, as far as they can provide a better understanding of this model. Usually, one considers separately the bosonic and fermionic versions of this model, as well as positive (repulsive) or negative (attractive) $U$ on-site interaction.

In this paper, I derive exact results that connect, for a general magnitude of the interaction term $U$, the repulsive and attractive model, in terms of their eigenspectra and quantum diffusion properties. It applies to both fermionic and bosonic models, in any dimension and for general filling, as far as the underlying structure is bipartite. There is already a long history of works devoted to using 
all sorts of symmetries that simplify the problem, some of which being intrinsic (like the $\mathrm{SU}(2)$ spin symmetry in the electron hamiltonian), others being related to geometric properties of the underlying lattice or graph (like the bipartiteness property), and/or to particular electron filling(electron-hole transformation at half filling) [5] [6. In section 2, a symmetry property of the spectrum is derived, whch is certainly already known (see the side remark in ref $[4$ ), although I could not find it previously published in the present form. To the best of my knowledge, the consequence on quantum diffusion (section 3) is original. In any case, the demonstrations are not technically difficult, but some results may appear, at first sight, rather counterintuitive. Let me first summarize these results in two steps

(i) it will first be shown that on a bi-partite structure, for general filling and dimension, the spectrum for positive $U$ is opposite to the spectrum for negative $U$. In other words, to any eigenenergy $E$ for one model, it corresponds (in a one-to-one manner) the opposite energy $(-E)$ for the other model. The relation between corresponding eigenstates is simple and will be explicited.

(ii) It is then possible to compare the time evolution, with the same initial state, for the two models. It will be proved that, for some class of initial conditions, these quantum evolutions cannot be distinguished.

It is this latter assertion which may appear at first sight as surprising. As the simplest example, consider the case of two electrons of opposite spins located on the same site of a one dimensional chain at $t=0$. If one computes the wave packet at any positive time, one finds that the weight (probability) of any ket in the tensor product basis is the same for $U<0$ and $U>0$. The physical reason for that should appear clearly below.

\section{A symmetry property for the eigenspectrum}

Let us first prove the first part about the eigenspectrum property. We shall first construct a representation space $\mathcal{E}$ for the many-particle Hilbert space, and map the many body problem onto a single-particle one in $\mathcal{E}$.

\subsection{Warm-up: the two-electron case}

To get familiar with this representation, let us describe the two-electron case for a one-dimensional chain. With two electrons of opposite spin, we consider oneparticule states $|l, \sigma\rangle$, where $l$ runs on the sites and $\sigma= \pm 1$ refers to the spin ,

which form a basis for the one-particle Hilbert space, and construct 2-particle states in the tensor product basis $\mathcal{B}$

$$
\mathcal{B}=\{|M\rangle=|l,+\rangle \otimes|m,-\rangle=|l, m\rangle, \quad l, m \in \mathbf{Z}\}
$$

The representation space $\mathcal{E}$ can safely be taken as a 2 dimensional square lattice, with a site $(l, m)$ associated with the above ket $|l, m\rangle$. Since each 
particle can hop only toward first neighbours in the chain, the non vanishing hamiltonian elements are restricted to first neighbours connections in $\mathcal{E}$, that is along the edges of the square lattice. The standard Hubbard hamiltonian

$$
H=\beta \sum_{<l m>, \sigma} c_{l \sigma}^{+} c_{m \sigma}+U \sum_{i} c_{l+}^{+} c_{l+} c_{l-}^{+} c_{l-}=T(\beta)+V(U),
$$

maps onto a one-particle standard tight-binding on a square lattice, with $\beta$ hopping terms, and a line of $U$ on-site potentials on the main diagonal,

$$
\begin{aligned}
& H=\beta \sum_{l m}(|l, m\rangle\langle l+1, m|+| l, m\rangle\langle l-1, m|+| l, m\rangle\langle l, m+1|+ \\
& |l, m\rangle\langle l, m-1|)+U \sum_{l}|l, l\rangle\langle l, l| .
\end{aligned}
$$

Note that, taking advantage of the periodicity along the diagonal (and constructing Bloch sums labelled by $k$ ), we can map this problem onto a family of one-dimensional problems with one impurity at the origin, and a $k$-dependant hopping term. The standard Slater-Koster sum rule for this impurity problem precisely corresponds to the Bethe-Ansatz condition for this two-electron problem [7].

Back to the 2 dimensional $\mathcal{E}$ space, we note the mirror symmetry along the diagonal, which allows to split the solutions into fully symmetrical and anti-symmetrical states, living repectively in $\mathcal{E}_{S}$ and $\mathcal{E}_{A}$. $\mathcal{E}_{S}$ is a half space, containing the diagonal, defined for instance as the points $\{(l, m), l \geq m\}$, a given such point representing the symmetrized ket $(|l, m\rangle+|m, l\rangle) / \sqrt{2} . \mathcal{E}_{A}$ is a also a half space, excluding the diagonal, defined as the points $\{(l, m), l>m\}$ , a given point representing the antisymmetrized ket $(|l, m\rangle-|m, l\rangle) / \sqrt{2}$. It is then immediately clear that the antisymmetrical sector is $U$-independent, as it is well known, and corresponds to the (addition) spectrum (without double occupancy) found in the problem of two electrons of equal spins. Indeed, in that case, Pauli principle imposes to restrict to the space antisymmetrical sector $\mathcal{E}_{A}$.

The main points of interest here is that the two-particle problems has been mapped onto a single particle problem in a larger space with a $U$ dependent subspace, and that the bipartiteness property of the original structure (the onedimensional chain) is shared by the larger space (either $\mathcal{E}, \mathcal{E}_{A}$ and $\mathcal{E}_{S}$ ). These properties remain for the general $N$-body problem, as we shall see now. Since spin is conserved in the Hubbard hamiltonian, the $N$-particle problem can be separated into independent problems where the number of particles of each spin is fixed. If particles live in a d-dimensional space $\mathcal{F}$, the large space $\mathcal{E}$ is just the euclidean sum of $N$ (a priori equivalent) copies of $\mathcal{F}$. The spectral problem can be first studied (as will be done below) in terms of a single particle problem in $\mathcal{E}$, selecting then among the eigensolutions those satisfying the symmetries associated with particle spins. An alternative way is to first reduce $\mathcal{E}$ by symmetry, and then solve the reduced hamiltonian. 


\subsection{Spectral symmetry}

Let us now derive our first result on the spectrum. We consider $\mathrm{N}$ particles, of specified spin, in a hypercubic lattice $Z^{d}$, and construct $N$-particle states $|M\rangle$, forming altogether a Hilbert space basis $\mathcal{B}$ represented by a $N d$ dimensional hypercubic lattice $\mathcal{E}$ :

$$
\mathcal{B}=\left\{|M\rangle=\bigotimes_{i=1, N}\left|r_{i}, \sigma_{i}\right\rangle\right\},
$$

More generally, if the one-particule states lives on a bi-partite graph $\Lambda$ (not necessarily hypercubic), with edges connecting kets with non vanishing hamiltonian matrix elements, so do the $N$-particule states. Indeed, let us first define a sign function $s(r)$, taking the value +1 or -1 according to which sub-lattice of $\Lambda, \vec{r}$ belongs. In the hypercubic lattice case with unit edge, $s(r)$ can for instance be defined as

$$
s(r)=(-1)^{\sum_{l=1, d} x_{l}}
$$

where $x_{l}$ are the $d$ coordinates of $\vec{r}$. The sign function $s(M)$ in the tensor product space is then simply defined as the product of the one-particule sign functions $s(r)$ entering the tensor product,

$$
s(M)=\prod_{i=1, N} s\left(r_{i}\right)
$$

Now, looking for non-vanishing elements $\left\langle M^{\prime}|H| M\right\rangle$, with $M^{\prime} \neq M$, it is clear that they occur only when one particule has jumped to a neighbouring site in $\Lambda$. This implies $s\left(M^{\prime}\right)=-s(M)$, which proves that the $N$-particule states live on a bipartite structure. The interacting part translates into $N(N-$ 1)/2 hyperplanes of dimension $(N d-1)$, with $U$ term as an on-site potential, corresponding to kets in $\mathcal{B}$ such that two particles share the same site in $\Lambda$. If these particles have equal spin, this manifold, that we shall call a $U$-hyperplane, will be eliminated by a proper antisymetrisation (which amount here to build new kets as the difference between kets that are symmetrically related by this hyperplane mirror). Note that iteration of this process vith the whole sets of hyperplanes for equal spins is nothing but constructing Slater determinant states. The $U$-hyperplanes intersect along hyperplanes of one dimension less, corresponding to states such that three particles meet on a $\Lambda$ site, and so on, down to a single "diagonal" $U$-space of dimension $d$, corresponding to states such that all particles share the same site. All these $U$-hyperplanes play a prominent role in the bosonic case, while only the one with highest dimension occurs for the electrons problem.

Since any symmetrization process done in $\mathcal{E}$ amounts to generate the proper combination of sites related by the $U$-hyperplane mirrors, it is not difficult to 
show that the $\mathcal{E}$ bi-partiteness property is shared by any of the properly symmetrized or anti-symmetrized sets corresponding to the particle statistics. Those willing to consider a specific type of particle statistics, or to use more standard Fock spaces and second quantised operators may prefer to redo the following computations in that framework, which will eventually turn quicker. But I prefer here to analyze the spectral properties in the (much) larger structure $\mathcal{E}$, to stress that the properties derived here ( on spectral symmetry and quantum diffusion) are statistics independent.

Let $\Delta(M)$ be the set of kets, different from $|M\rangle$, and connected to it by $H$,

$$
\Delta(M)=\left\{\left|M^{\prime}\right\rangle \in \mathcal{B}, M^{\prime} \neq M, \text { and }\left\langle M^{\prime}|H| M\right\rangle \neq 0\right\},
$$

and let $|\Psi(E,+)\rangle$ be an eigenket of $H(+|U|)$, with eigenenergy $E$, whose decomposition reads

$$
|\Psi(E,+)\rangle=\sum_{M \in \mathcal{B}} a_{M}^{E}|M\rangle
$$

By projecting the Schrödinger equation onto the set of bra $\langle M|$, one gets a set of secular equations

$$
\beta \sum_{M^{\prime} \in \Delta(M)} a_{M^{\prime}}^{E}=(E-f(M, U)) a_{M}^{E},
$$

where $f(M, U)=\langle M|H| M\rangle$ is related to the number of multiply occupied 1-particle orbitals in $|M\rangle$. The only property which we now use is that $f$ is an odd function of $U$ (it is in fact a number time $U$ ). Let us now consider the ket

$$
\left|\Psi^{\prime}\right\rangle=\sum_{M \in \mathcal{B}} s(M) a_{M}^{E}|M\rangle
$$

where $s(M)$ is the above defined sign function. Since $M$ and $\Delta(M)$ live on two different $\mathcal{E}$ subparts (for any $M$ ), $\left|\Psi^{\prime}\right\rangle$ clearly satisfies the set of secular equations

$$
\beta \sum_{M^{\prime} \in \Delta(M)} a_{M^{\prime}}^{E}=(-E-f(M,-U)) a_{M}^{E}
$$

which proves that $\left|\Psi^{\prime}\right\rangle$ is an eigenstate of $H(-|U|)$, with $-E$ as its eigenvalue: $\left|\Psi^{\prime}\right\rangle=|\Psi(-E,-)\rangle$. This correspondance can be carried out for all eigenvalues in the spectrum, and we can write (Sp denoting the set of eigenvalues in the spectrum)

$$
\mathbf{S p}(H, U) \equiv-\mathbf{S p}(H,-U)
$$

At this point, two remarks are worth pointing out

(i) If one consider only changing the hopping term sign, one easily finds (a well known result) that

$$
\mathbf{S p}(H,-\beta, U) \equiv \mathbf{S p}(H, \beta, U)
$$


with, to a given eigenvalue $E,|\Psi\rangle$ as an eigenket for $H(\beta, U)$ and $\left|\Psi^{\prime}\right\rangle$ for $H(-\beta, U)$

(ii) The hamiltonian $H$ is the sum of a kinetic part $T(\beta)$ and an interacting part $V(U)$. In the $\mathcal{E}$ space, as said above, this $V(U)$ part is an on-site interaction, like in impurity models, whose value depends on the particular tensor product kets. The kinetic part takes a constant value whenever two kets are connected by $H$. This reminds of the one-electron so-called Anderson hamiltonian, but with two major difference:

- In the Anderson hamiltonian, the diagonal terms are randomly distributed, while here the $U$ dependant terms are heavily correlated.

- One usually studies this Anderson hamiltonian onto lattices of physocal interest (in dimension 1,2 or 3 ), why $\mathcal{E}$ is of high dimension.

It is nevertheless worth noticing that the above symmetry on the two spectra apply as well to a standard Anderson hamiltonian, whenever all the random diagonal terms have their sign changed simultaneously. At this point, one should recall some recent work done on the two-electron problem (TIP) with Hubbard hamiltonian, in the context of possible interaction induced delocalization [8]. If the (random, diagonal) potential distribution law is symmetrical around zero, the properties of the model ( like the eigenstates localization length) should not change if all diagonal terms have their sign changed. In such a case, one can predict that the interaction induced delocalization around a given energy $E$ (with Hubbard term $U$ ) should be identical to what occurs for $-E$ and $-U$

\section{Quantum Diffusion}

We now compare quantum diffusion (evolution under the time-dependant Schrödinger equation) for both sign of $U$ (attractive or repulsive interaction), and with specified initial conditions. We first study the simplest case, where $|\Psi(t=0)\rangle=$ $|M\rangle \in \mathcal{B}$. As usual, the time evolution is computed by expanding $|M\rangle$ in the complete set of eigenkets $\mathcal{E}_{ \pm}=\{|\Psi(E, \pm)\rangle\}$. Let $W$ (inverse of the above introduced $a_{M}^{E}$ ) be the unitary matrix transforming the tensor product basis $\mathcal{B}$ into the eigenket basis $\mathcal{E}_{+}$. Let us stress that $H$ being a real hamiltonian (which for instance would not be the case in the presence of a magnetic field), it is always possible to take $W$ as a real matrix, which is now assumed. Now, with $D=\operatorname{Card}(\mathcal{B})$, we can write

$$
\begin{aligned}
|\Psi(t=0)\rangle & =|M\rangle=\sum_{l=1}^{D} W_{l}^{M}\left|\Psi_{l}\right\rangle, \text { and } \\
|\Psi(t)\rangle & =\sum_{l=1}^{D} W_{l}^{M} \exp \left(-i E_{l} t / \hbar\right)\left|\Psi_{l}\right\rangle
\end{aligned}
$$

where $l$ labels the set of eigenstates of $H(|U|)$. The componant of $|\Psi(t)\rangle$ on 
the ket $\left|M^{\prime}\right\rangle \in \mathcal{B}$ simply reads

$$
\left\langle M^{\prime} \mid \Psi(t)\right\rangle=\sum_{l=1}^{D}\left(W_{l}^{M^{\prime}}\right)^{T} W_{l}^{M} \exp \left(-i E_{l} t / \hbar\right)
$$

Now, with the same initial condition, let us compute the corresponding ket at time $t,\left|\Psi^{-}(t)\right\rangle$, for the hamiltonian $H(-|U|)$. With the above discussed relations between the eigenvalues and eigenkets, one easily obtains

$$
\left\langle M^{\prime} \mid \Psi^{-}(t)\right\rangle=s(M) s\left(M^{\prime}\right) \sum_{l=1}^{D}\left(W_{l}^{M^{\prime}}\right)^{T} W_{l}^{M} \exp \left(i E_{l} t / \hbar\right) .
$$

Note that the product $s(M) s\left(M^{\prime}\right)$ takes only two values, +1 if $M$ and $M^{\prime}$ belong to the same sublattice, and -1 otherwise. So, in any case, we find a simple correspondance between $|\Psi(t)\rangle$ and $\left|\Psi^{-}(-t)\right\rangle$,

$$
\left\langle M^{\prime} \mid \Psi(t)\right\rangle= \pm\left\langle M^{\prime} \mid \Psi^{-}(-t)\right\rangle
$$

Comparing both evolution for positive time, it comes

$$
\begin{aligned}
\left|\left\langle M^{\prime} \mid \Psi^{-}(t)\right\rangle\right|^{2} & =\left(\sum_{l=1}^{D}\left(W_{l}^{M^{\prime}}\right)^{T} W_{l}^{M} \exp \left(i E_{l} t / \hbar\right)\right) \times \\
& \left(\sum_{l=1}^{D}\left(W_{l}^{M^{\prime}}\right)^{T} W_{l}^{M} \exp \left(-i E_{l} t / \hbar\right)\right) \\
& =\left|\left\langle M^{\prime} \mid \Psi(t)\right\rangle\right|^{2}
\end{aligned}
$$

So quantum diffusion, at any time $t$, is identical (in terms of probabilities with respect to any ket in the basis $\mathcal{B}$ ), for repulsive and attractive interaction of the same magnitude $(|U|)$. This (surprising?) result is thus proved for any basis ket $|M\rangle$, taken as an initial condition. It can be understood, on physical grounds, by recalling first that quantum diffusion is done at constant energy. The initial ket is delocalized in energy and its time evolution is influenced by low and high energy eigenket contributions. So, states close to the ground state energy for attractive $U$ correspond to more or less gathered particles, while states with higher energy, eventhough we are in an attractive $U$ model, correspond to more separated particles (this recalls the difference between standard bonding and antibonding states for one-electron states). So high energy states for attractive $U$ influences the quantum evolution in a similar manner as low energy states for repulsive $U$, which qualitatively explain the above result.

We now consider a more generic initial conditions for the quantum diffusion. Let us split $\mathcal{E}$ (and therefore $\mathcal{B}$ ) into two parts $\mathcal{E}_{A}$ and $\mathcal{E}_{B}$, according to the bi-partite decomposition, (corresponding to a basis partition $\mathcal{B}_{A}$ and $\mathcal{B}_{B}$ ), and write

$$
|\Psi(t=0)\rangle=\sum_{M \in \mathcal{B}_{A}} b_{M, A}|M\rangle+\sum_{M \in \mathcal{B}_{B}} b_{M, B}|M\rangle
$$


It is then easy to write the components of $|\Psi(t)\rangle$ in the basis $\mathcal{B}$ :

$$
\left\langle M^{\prime} \mid \Psi(t)\right\rangle=\sum_{l=1}^{D}\left(W_{l}^{M^{\prime}}\right)^{T} \exp \left(-i E_{l} t / \hbar\right)\left[\sum_{M \in \mathcal{B}_{A}} b_{M, A} W_{l}^{M}+\sum_{M \in \mathcal{B}_{B}} b_{M, B} W_{l}^{M}\right] .
$$

The components of $\left|\Psi^{-}(t)\right\rangle$ read accordingly

$$
\begin{aligned}
\left\langle M^{\prime} \mid \Psi^{-}(t)\right\rangle & =s\left(M^{\prime}\right) \sum_{l=1}^{D}\left(W_{l}^{M^{\prime}}\right)^{T} \exp \left(i E_{l} t / \hbar\right) \times \\
& {\left[s(A) \sum_{M \in \mathcal{B}_{A}} b_{M, A} W_{l}^{M}+s(B) \sum_{M \in \mathcal{B}_{B}} b_{M, B} W_{l}^{M}\right] }
\end{aligned}
$$

It is clear that now, $\left|\left\langle M^{\prime} \mid \Psi^{-}(t)\right\rangle\right|^{2}=\left|\left\langle M^{\prime} \mid \Psi(t)\right\rangle\right|^{2}$ only if the initial ket $|\Psi(t=0)\rangle$ lives on one sub-structure only (either $A$ or $B$ ), with all components $b_{M}$ in phase. This restricts the choice of initial conditions for which this $(U,-U)$ parallel behaviour occurs to a large but definitively non generic set. Note however that one is often inclined, owing to the linearity of Schrödinger equation, to restrict the numerical studies of quantum evolution to basis kets as initial conditions, which precisely belong to this non generic category.

Acknowledgements: It is a pleasure to thank Claude Aslangul, Michel Caffarel, Benoit Douçot and Julien Vidal for fruitful discussions.

\section{References}

[1] Hubbard J., Proc. Roy. Soc. London, A277, 237

[2] E.H. Lieb and F.Y. Wu, Phys. Rev. Lett., 20, 1445 (1968)

[3] E. Dagotto, Rev. Mod. Phys. 66, 763 (1994).

[4] B. Sutherland in "Exactkly Solvable Problems in Condensed Matter and Relativistic Field TheoryThe Hubbard model", Lecture Notes in Physics 242 Springer-Verlag 1985

[5] E.H. Lieb, in "The physics and mathematical physics of the Hubbard model", NATO Workshop, 1993 Plenum Press

[6] A. Auerbach, "Interacting electrons and quantum magnetism", Springer Verlag, New York, 1994).

[7] M. Caffarel and R. Mosseri, Phys. Rev. B , 57, R12651 (1998)

[8] D.L. Shepelyansky, Phys.Rev.lett., 73, 2607 (1994) 